\section{European Urology}

\section{J. Curtis Nickel}

Department of Urology, Queen's University, Kingston General Hospital, Kingston, Ont., Canada

\title{
Clinical Guidelines for Prostatitis: Are We Ready Yet?
}

In this month's issue of European Urology, an impressive group of academic urologists representing a cross section of European countries, report on a consensus meeting relating to the important topic of management of bacterial prostatitis. They have written an excellent review, provided a very thorough, somewhat opinionated analysis of the data they evaluated and have developed a set of 'practical guidelines' for the clinical management of chronic bacterial prostatitis. And herein lies the problem.

I am concerned about the many "clinical practice guidelines' which are metastasizing throughout the medical literature, not only in urology, but in all fields of disease management. Are such "practice management guidelines' a bad thing for medicine? Not necessarily. They can be extremely useful for clinicians, primarily those who are not experts in the disease in question, in guiding the management of many of their patients presenting with the specific complaint. But for guidelines to be useful they must be developed following a very strict protocol. Guidelines developed for benign prostatic hyperplasia by the International BPH Consultation and the US Department of Public Health are ideal models to follow.

But these guidelines presented for the management of chronic bacterial prostatitis are problematic. The authors are respected thought leaders, their credentials are above reproach, their opinions are to be highly valued, yet I disagree with many of their conclusions (which is okay) and some of their recommendations which are now described as guidelines (which may not be okay). I could bring together a similar group of prostatitis 'experts', review the same literature and perhaps come to altogether different conclusions and recommendations.

\begin{tabular}{ll}
\hline KARGER & ( 1998 S. Karger AG, Basel \\
Fax + 41 61306 12 34 & 0302-2838/98/0346-0455\$15.00/0 \\
$\begin{array}{l}\text { E-Mail karger@karger.ch } \\
\text { www.karger.com }\end{array}$ & $\begin{array}{l}\text { Accessible online at: } \\
\text { http://BioMedNet.com/karger }\end{array}$
\end{tabular}

In my opinion, any guidelines published for the management of prostatitis should include all the following considerations:

(1) A defined process developed prior to any meeting to decide who should be on the panel and who should be represented (i.e. by country, organization, academic standing, etc.).

(2) An independent systemic search of the world literature, with established criteria for selection of studies to be considered for the guidelines and an indication of how the scientific merit of the studies were assessed.

(3) A predefined process by which the accumulated literature would be audited and reviewed in an unbiased manner.

(4) A predefined process by which recommendations would be made.

(5) An indication of how strong the evidence is for a particular recommendation.

(6) An estimate of how large an impact the recommendations will have if universally implemented.

(7) An independent peer review of the recommendations.

(8) An opportunity for the sponsoring countries, organizations, etc. to have input prior to publication of the guidelines.

(9) No direct financial association with any 'interested' pharmaceutical company.

(10) A process by which the implementation of the recommendations would be audited.

(11) A process under which the guidelines would be reviewed and updated.
J. Curtis Nickel, MD
Department of Urology, Queen's University
Kingston General Hospital
Kingston, Ont. K7L 2V7 (Canada)
Tel. +1 613548 2497, Fax +1 6135451970 
The authors have (perhaps very wisely) chosen a very small percentage of prostatitis patients (i.e. chronic bacterial prostatitis) to develop their guidelines. Hypothetical$1 y$, it would seem that recommendations for the approximately $5 \%$ of prostatitis patients who have positive prostate localization cultures with uropathogens would be simple and noncontroversial. The authors, in fact, have listed some of the very real limitations in developing guidelines for this particular disease. A number of specific examples are listed:

(1) Although many papers describe the differential leukocytosis present in the expressed prostatitic fluid of prostatitis patients, there has never been adequate validation that this is useful or even meaningful in the differentiation of NIH Category II (chronic bacterial) and Category IIIA (nonbacterial) prostatitis, or for that matter, with Category IIIB (so-called prostatodynia).

(2) The relevance of negative cultures of expressed prostatic fluid, especially in previously treated patients, has never been validated. In fact, much of the recent literature looking at the etiologic basis for this disease employing sophisticated culture, immunological and molecular biological techniques have suggested that cryptic nonculturable microorganisms might be implicated. Some feel that repetitive prostate massage will reveal the pathogenic organism, when a single expressed prostatic secretion (EPS) culture may fail to do so.

(3) The literature does not convincingly demonstrate that eradication of uropathogenic organisms from the EPS results in long-term cure of the prostatitis. In fact, the average practicing urologist will attest to exactly the opposite.

(4) The authors recommend a 2-week trial of empiric antibiotic therapy regardless of culture results. Nowhere in the literature can this be validated as appropriate. In fact, most clinical trials employing empiric therapy, employ it for 4-6 weeks. The recent report (AUA, San Diego, June 1998) from our Canadian Prostatitis Research Group showed that even a 4-week course of empiric antibiotic therapy did not predict the final outcome of 12 weeks of antibiotics (for both culture-negative and culture-positive cases).

The authors recommend clinical studies. The specific studies recommended are certainly important, but perhaps premature. The most important considerations in advancing the knowledge and subsequent management of the prostatitis diseases are, in this author's opinion (and some of these considerations are actually raised by the authors as well):
(1) Development of a validated symptom score, so that response can be reliably and consistently evaluated within and between treatment groups as well as between studies.

(2) Determination of the true epidemiological importance of this disease in terms of incidence, prevalence, quality-of-life issues and costs incurred by patients and society with properly designed population-based studies.

(3) Determination and validation of the true importance of EPS leukocytosis.

(4) Validation of the true importance of positive and negative EPS cultures.

(5) Determination of the long-term outcome of antibiotic therapy in prostatitis.

I would like to end this commentary the way I started it. By commending the authors on an excellent review of the literature and development of their consensus management statement based on personal interpretation and clinical experience in this difficult and frustrating disease. Are we ready for publication of guidelines for the management of prostatitis? I think not! Do we need the type of recommendations published in this month's European Urology to help guide us through the clinical quagmire of prostatitis. Absolutely! 